\title{
Massives Hirnödem im Rahmen eines posterioren reversiblen Enzephalopathiesyndroms (PRES)
}

\section{Massive Cerebral Edema in the Course of a Posterior Reversible Encephalopathy Syndrome (PRES)}

Autoren

Institute
J. Schaumberg ${ }^{1}$, D. Schwandt ${ }^{1}$, P. Michels ${ }^{1}$, F. Kämmerer ${ }^{2}$, B. Eckert ${ }^{2}$, J. Röther ${ }^{1}$

${ }^{1}$ Klinik für Neurologie, Asklepios Klinik Altona, Hamburg

${ }^{2}$ Klinik für Neuroradiologie, Asklepios Klinik Altona, Hamburg

\section{Schlüsselwörter \\ - PRES \\ - Hirnödem \\ - Enzephalopathie \\ - Bluthochdruck \\ Key words \\ - PRES \\ - RPLS \\ - encephalopathy \\ - cerebral edema \\ - high blood pressure}

\section{Bibliografie}

DOI http://dx.doi.org/ 10.1055/s-0034-1382031 Akt Neurol 2014; 41: 356-359 (c) Georg Thieme Verlag KG Stuttgart · New York ISSN 0302-4350

Korrespondenzadresse Dr. Jens Schaumberg Neurologie

Asklepiosklinik Altona Paul-Ehrlich-Straße 1 22765 Hamburg j.schaumberg@web.de

\section{Zusammenfassung}

$\nabla$

Der Fallbericht zeigt einen ungewöhnlichen Verlauf eines PRES bei einer 49-jährigen Frau mit akuter Vigilanzminderung bei massiv ausgeprägtem vasogenen Hirnödem. Unter einer konsequenten antihypertensiven Therapie kam es klinisch und bildmorphologisch zu einer deutlichen Besserung. Das Fallbeispiel beschreibt die klinische Manifestation und Therapie des PRES im Kontext der aktuellen Literatur.

\section{Einleitung}

Das posteriore reversible Enzephalopathiesyndrom (PRES) wurde erstmalig von Hinchey und Kollegen 1996 beschrieben und ist durch Symptome wie Kopfschmerzen, Übelkeit, Desorientiertheit, epileptische Anfälle oder Sehstörungen in Kombination mit typischen, meist reversiblen bildgebenden Veränderungen eines subkortikalen vasogenen Ödems mit Prädilektionsstellen parietal und occipital gekennzeichnet [1]. Aufgrund atypischer Manifestationen mit Läsionen in den frontalen oder temporalen Hirnabschnitten sowie einer gelegentlichen kortikalen Mitbeteiligung werden z.T. allgemein gehaltene Synonyme wie reversible Leukenzephalopathie oder hypertensive Enzephalopathie benutzt [2,3]. Die genaue Inzidenz oder Prävalenz der Erkrankung ebenso wie die Pathophysiologie der Erkrankung sind unklar. Gängige Vorstellungen der Entstehung des vasogenen Ödems reichen von der Theorie der zerebralen Hyperperfusion im Rahmen von hypertensiven Krisen mit Dekompensation der zerebralen Autoregulation hin zur Vorstellung einer zerebralen Hypoperfusion im Rahmen einer zerebralen Vasokonstriktion. Alternativ wird eine endotheliale Dysfunktion der Gefäße durch Toxine bei z.B. Sepsis, Autoimmunerkran-

\section{Abstract \\ $\nabla$}

We report here on an unusual case of posterior reversible encephalopathy syndrome (PRES) in a 49-year-old woman with who suffered an acute episode of somnolence caused by massive vasogenic cerebral edema. Under intravenous antihypertensive therapy, there was significant clinical improvement associated with regressive MRI findings. This case describes the clinical manifestation and therapy of PRES in the context of the current literature.

kungen, Kontrastmittelgabe und Präklampsie diskutiert ( $\bullet$ Tab. 1) $[2,4]$.

\section{Kasuistik}

$\nabla$

Die Aufnahme einer 49-jährigen Frau erfolgte aufgrund einer akuten Vigilanzminderung. Fremdanamnestisch sei sie zuvor wesensverändert und lethargisch gewesen und habe über starke Kopfschmerzen geklagt. Bis auf eine Präklampsie vor 18 Jahren waren keine Vorerkrankungen bekannt.

\section{Neurologischer Befund}

Bei der Aufnahme zeigte sich eine somnolente, zwischenzeitlich auch agitierte Patientin mit gezielter Abwehr aller Extremitäten auf Schmerzreiz. Keine sprachlichen Äußerungen, Aufforderungen kam die Patientin nicht nach, keine pathologischen Reflexe. Zudem fand sich eine neu aufgetretene Abducensparese links, eine homonyme Hemianopsie nach links sowie ein Strabismus convergens. Zum Aufnahmezeitpunkt bestand eine hypertensive Krise mit systolischen Blutdruckwerten von $250 \mathrm{mmHg}$. 
Tab. 1 Auswahl an häufigen Ursachen des PRES, modifiziert nach [2].

\begin{tabular}{|ll}
\hline häufige Ursachen des PRES & Besonderheiten \\
\hline arterielle Hypertonie & meist akute Dekompensation \\
\hline $\begin{array}{l}\text { Nierenfunktionsstörung } \\
\text { Präklampsie/Eklampsie }\end{array}$ & akut oder toxisch \\
\hline immunsuppressive Medikamente & oft Erstgebärende \\
\hline Transplantation & $\begin{array}{l}\text { hä. Calcineurinhemmer (z. B. Tacrolimus), Chemotherapeutika (z. B. Cisplatin, Gemcitabine, Bevazizumab) } \\
\text { immunsuppressiver Therapie }\end{array}$ \\
\hline $\begin{array}{l}\text { Autoimmunerkrankungen } \\
\text { Infektionen }\end{array}$ & $\begin{array}{l}\text { z. B. systemischer Lupus Erythematodes, M. Wegener, Polyarthritis nodosa, Psoriasis, M. Basedow } \\
\text { häufiger bei grampositiven Erregern }\end{array}$ \\
\hline
\end{tabular}
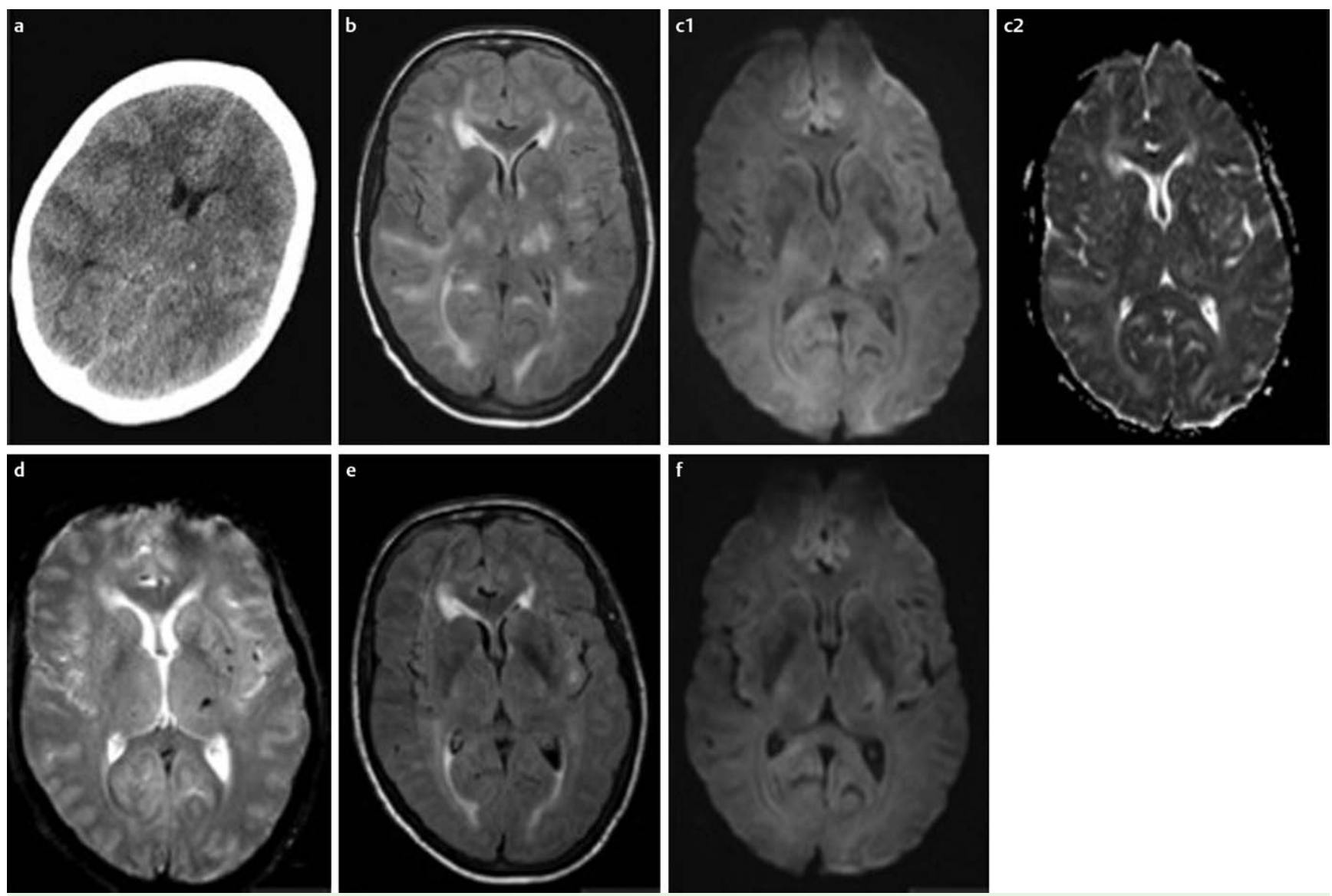

Abb. 1 a Initiales CCT mit ausgeprägter Leukenzephalopathie und massivem Hirnödem mit nahezu aufgebrauchten Liquorreserveräumen und Darstellung einer Mikroblutung im Thalamus links. Die Mark-Rinden-Grenze kann differenziert werden. b cMRT mit fluid-attenuated inversion recovery (FLAIR) - Wichtung 2 Tage nach Aufnahme mit flächenhaften, subkortikalen Marklagerödem ohne Diffusionsstörung c1 Die Thalamusblutung links zeigt sich hypointens in der DWI. c2 Korrespondierendes ADC-Parameter-

\section{Zusatzbefunde}

\section{$\nabla$}

Die initiale kranielle Computertomografie (cCT) zeigte eine ausgeprägte Leukenzephalopathie mit massivem Hirnödem ( $\bullet$ Abb. 1a) mit einer kleinen Mikroblutung im Thalamus links ohne Schrankenstörung oder Hinweise auf einen Hirninfarkt. Laborchemisch fielen erhöhte Nierenretentionswerte mit einem Kreatinin von $2,1 \mathrm{mg} / \mathrm{dl}(0,6-1,0 \mathrm{mg} / \mathrm{dl})$ und ein erhöhtes Troponin I mit $281 \mathrm{ng} / \mathrm{l}(<30 \mathrm{ng} / \mathrm{l})$ auf. In der Liquordiagnostik im Verlauf zeigte sich bei normaler Zellzahl ein erhöhtes Gesamteiweiß von $994 \mathrm{mg} / \mathrm{l}(<500 \mathrm{mg} / \mathrm{l})$ und eine Schrankenstörung.

bild (apparent diffusion coefficient) mit beschleunigter Diffusion (hyperintense Areale) als Ausdruck des vasogenen Ödems d cMRT im Verlauf mit echo planar T2 - Wichtung und Darstellung von weiteren linksbetonten Mikroblutungen e cMRT im Verlauf mit FLAIR - und Diffusionswichtung $\mathbf{f}$ im Vergleich zum vorherigen cCT und cMRT mit Rückbildung des Hirnödems und deutlicher Rückbildung der Marklagerläsionen.

\section{Verlauf}

$\nabla$

Die Patientin wurde bei weiter wechselnder Vigilanz auf unsere Neurologische Überwachungsstation aufgenommen und bei Verdacht auf einen non konvulsiven Status epilepticus eine antikonvulsive Medikation mit Levetiracetam eingeleitet. Der Blutdruck war weiter labil und konnte nur mittels Clonidin- und Urapidil-Perfusoren auf systolische Spitzendrücke bis maximal $160 \mathrm{mmHg}$ eingestellt werden. Im Verlauf kam es unter konsequenter Blutdruckeinstellung innerhalb von $24 \mathrm{~h}$ zu einer langsamen Reorientierung der Patientin. Bildmorphologisch zeigte sich im cMRT am dritten stationären Tag eine deutliche, im 


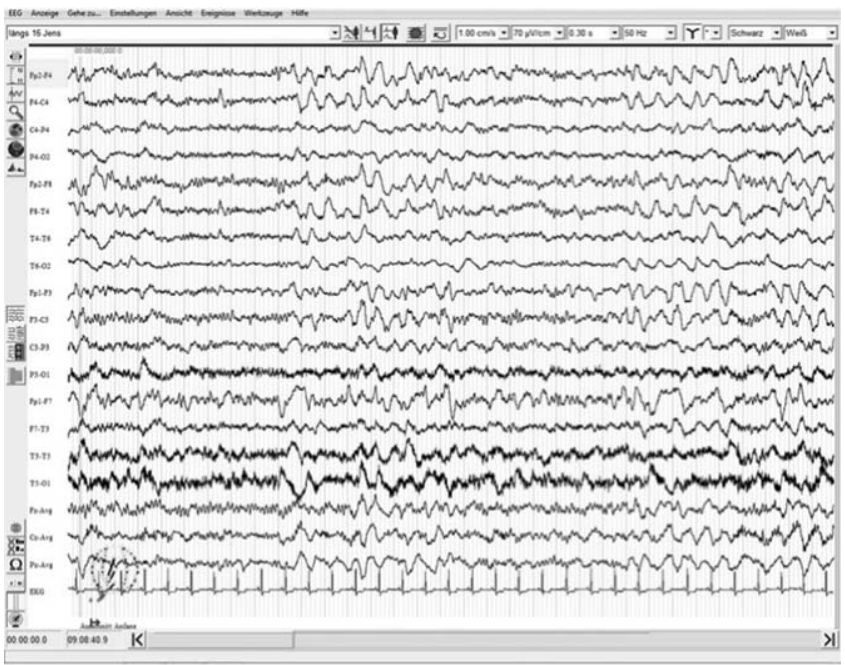

Abb. 2 EEG in bipolarer Längsreihe mit mittelgradiger Allgemeinveränderung und einer intermittierenden rhythmischen Deltaaktivität (IRDA).

Vergleich zur cCT aber insgesamt rückläufige Leukenzephalopathie mit regredientem Hirnödem ohne Diffusionsstörungen ( $\bullet$ Abb. 1b c1, c2). Im EEG stellte sich eine mittelgradige Allgemeinveränderung sowie Zeichen einer subkortikalen Funktionsstörung ( $\bullet$ Abb. 2) dar. Die beginnende Niereninsuffizienz war unter forcierter Diurese rückläufig. Hinweise auf eine zugrunde liegende Nierenarterienstenose fanden sich nicht. Zusätzlich zeigte sich kein Hinweis auf eine endokrine, rheumatologische, toxische oder kardiale Erkrankung als Ursache einer sekundären Hypertonie. In der Untersuchung des Augenhintergrunds stellte sich ein Fundus hypertonicus $\mathrm{IV}^{\circ}$ mit geringen Einblutungen dar. In der Verlaufsbildgebung mittels cMRT zeigte sich im Vergleich eine weiter rückläufige Leukenzephalopathie ( $\bullet$ Abbs. 1d, e, f). Die Patientin konnte nach 12 Tagen in klinisch neurologisch unauffälligem Status selbstständig nach Hause entlassen werden.

\section{Diskussion}

\section{$\nabla$}

Wir beschreiben den klinischen und bildmorphologischen Verlauf eines PRES bei einer 49-jährigen Frau. Ungewöhnlich an unserem Fallbeispiel ist das massiv ausgeprägte vasogene Hirnödem mit aufgebrauchten Liquorräumen und Hirndruckzeichen. Diese seltene Verlaufsform des PRES ist in Einzelfällen beschrieben und kann einen fatalen Verlauf nehmen $[5,6]$. Wichtig ist die differenzialdiagnostische Einordnung der klinischen und bildmorphologischen Befunde mit unmittelbarer Einleitung einer aggressiven intensivmedizinischen antihypertensiven sowie ggf. antiödematösen Therapie [6]. Dabei kann eine intrakranielle Druckmessung im Einzelfall sinnvoll sein [7]. Klinisch manifestiert sich die Erkrankung initial durch Kopfschmerzen, Sehstörungen, Verwirrtheitszustände, Übelkeit oder epileptische Anfälle. Die Symptome entwickeln sich meist innerhalb von Stunden und bilden sich in einem Zeitraum von einer bis selten mehreren Wochen zurück. Es werden aber auch irreversible und atypische Verläufe beschrieben [2,3]. Kinder und Erwachsene können betroffen sein [8]. Selten kommt es zu Prodromalsymptomen wie einer allgemeinen Erschöpfung, Müdigkeit oder Schmerzen [2].
Die Diagnose kann initial, wie in unserem Fall, schwierig sein, da PRES-typische Läsionen in nur ca. 50\% der Fälle im cCT dargestellt werden können [2].

Bildgebung: Typischerweise finden sich im cCT flächige Hypodensitäten occipital beidseits. Dabei kann die Differenzierung zu ischämischen Läsionen problematisch sein, da die Mark-Rinden-Grenze nicht immer sicher beurteilbar ist. Die bei PRESLäsionen nicht eingehaltenen territorialen Grenzen der Gefäßversorgungen können zur Differenzierung hilfreich sein $[1,2]$. Domäne für den PRES-Nachweis bleibt die CMRT, die bei Verdacht auf, PRES möglichst rasch anzustreben ist. In der cMRT stellt sich das PRES typischerweise mit Hyperintensitäten in den T2- und in den fluid-attenuated inversion recovery (FLAIR) gewichteten Sequenzen in den parieto-occipitalen Regionen dar $[4,9]$. Dabei zeigt sich charakteristisch eine relativ symmetrische, flächige Schädigung im subkortikalen Marklager und partiell auch im angrenzenden Kortex [1,2,9,10]. Einseitige Läsionsmuster sowie ein isoliertes Auftreten von atypischen Läsionsmustern stellen die Ausnahme dar [2,9,11]. Bei einem Drittel der Patienten können Läsionen in den Basalganglien, Kleinhirn oder Hirnstamm gefunden werden [1,2]. Einblutungen stellen sich am besten in der „echo planar T2*“ oder der suszeptibilitätsgewichteten (SWI) Sequenz dar. Dabei scheint das Auftreten der Blutung unabhängig vom Blutdruck zu sein. Vielmehr scheinen Gerinnungsstörungen, Transplantationen oder eine Immunsuppression eine Rolle zu spielen. [2,12]. Es kommen intraparenchymatöse sowie meist kortikale subarachnoidale Blutungen mit einer Inzidenz von 15\% bei Patienten mit PRES vor. [12] Die diffusionsgewichtete Epi-Sequenz und dabei insbesondere der apparent diffusion coefficient (ADC) kann Hinweise zur Differenzierung zwischen einem vasogenen oder zytotoxischen Ödem geben. Ein vasogenes Ödem ist das typische Bild bei einem PRES. Zytotoxische Ödeme können im Rahmen eines PRES auftreten, sind jedoch nicht „klassisch“ mit einem PRES vergesellschaftet. Das zytotoxische Ödem, wie z.B. beim ischämischen Schlaganfall zeigt sich in der DWI-Sequenz deutlich hyperintens mit einer Signalabsenkung in der korrespondierenden ADC-Wichtung. Im Gegensatz dazu zeigt sich ein vasogenes Ödem in der Regel in der DWI-Wichtung isointens bis minimal hyperintens bei fehlender Signalabsenkung in der ADC-Wichtung [2,3,9]. Eine Kontrastmittelgabe führt in der Regel zu keinem Informationsgewinn $[3,9]$. Der beste Zeitpunkt zur weiteren Verlaufsuntersuchung und Darstellung der Reversibilität ist unklar [9]. Er liegt im Allgemeinen zwischen einigen Tagen und mehreren Wochen und kann im Bedarfsfall wiederholte bildgebende Untersuchungen mittels cMRT notwendig machen.

Einige Fälle von rezidivierenden PRES Episoden sind beschrieben [9].

EEG: Im EEG finden sich unspezifische Veränderungen mit einer diffusen Verlangsamung und fokalen Delta-Herden. Bei Patienten mit einem Status Epilepticus und PRES finden sich meist rhythmische Deltawellen und sharp-wave-Komplexe in parietooccipitaler Lokalisation, wobei häufig fokale Anfallsstatus mit sekundärer Generalisation auftreten [2,13]. Die Labordiagnostik inklusive Liquor ist unspezifisch und dient der Ausschluss- bzw. Differenzialdiagnostik. Bei Bestehen eines Hirnödems kann eine Lumbalpuktion initial kontraindiziert sein. Der Nachweis von hypertensiv bedingten Endorganschäden kann die ätiologische Zuordnung vereinfachen. In unserem Fallbeispiel gehen wir aufgrund der Endorganschäden der Niere, der Augen und der anamnestisch bestehenden Praeklampsie von einer dekompensierten vorbestehenden arteriellen Hypertonie als Ursache des PRES 
aus. Zu den Differenzialdiagnosen des PRES zählen das reversible zerebrale Vasokonstriktionssyndrom, der ischämische Schlaganfall v.a. im hinteren Stromgebiet, die Enzephalitis, eine Sinusvenenthrombose und die primäre zerebrale Vaskulitis [2]. Gerade die initiale Abgrenzung zum reversiblen zerebralen Vasokonstriktionssyndrom ist schwierig. Dieses beginnt oft mit einem Donnerschlag-Kopfschmerz und entwickelt sich klinisch langsamer. In der Angiografie zeigen sich „string of beads“-Muster, welche jedoch selten auch beim PRES vorkommen können. Die weitere Abgrenzung bringt die Bildgebung mittels cMRT, da beim reversiblen Vasokonstriktionssyndrom in der Regel keine bilateralen parieto-occipitalen Läsionen wie beim PRES auftreten [2].

Die Therapie der arteriellen Hypertonie mit intravenösen Antihypertensiva steht beim PRES im Vordergrund. Roth und Kollegen empfehlen dabei einen systolischen Blutdruck von unter $160 \mathrm{mmHg}$, welcher bei unserer Patientin nur durch 2 kontinuierlich intravenös applizierte Antihypertensiva erreicht werden konnte. Eine Behandlung mit Nitroglycerin scheint das Krankheitsbild eher zu verschlechtern und sollte nicht als Medikament der ersten Wahl eingesetzt werden. Die Rolle von Corticosteroiden ist unklar. Beim Auftreten von epileptischen Anfällen sollten diese mit Antikonvulsiva behandelt werden. Dabei scheint ein Zeitraum von 3 Monaten für die antikonvulsive Medikation bei nachfolgender Anfallsfreiheit und fehlenden epilepsietypischen Aktivitäten im EEG ausreichend [2,13].

Zusammenfassend zeigt der Fallbericht eine äußerst schwere Form des PRES mit massivem Hirnödem und Hirndruckzeichen mit regredientem Verlauf unter intravenöser antihypertensiver Therapie. Die schnelle Einordnung der Symptome und eine konsequente, aggressive Einstellung des Blutdrucks sind für die erfolgreiche Therapie entscheidend. In unserem Fall spielte die Blutdruckentgleisung mit nachfolgendem „breakthrough of autoregulation" sicher eine entscheidende Rolle [8,14]. Es bedarf weiterer Studien um zu klären, welche pathophysiologische Grundlagen sonst noch dem klinischen Bild des PRES ohne arterieller Hypertonie zugrunde liegen und ob diese unterschiedliche Therapien nach sich ziehen.

\section{Interessenkonflikt}

\section{$\nabla$}

Die Autoren geben an, dass kein Interessenkonflikt besteht.

\section{Literatur}

1 Hinchey J, Chaves C, Appignani B et al. A Reversible posterior leukencephalopathy Syndrom. NEJM 1996; 34: 494-500

2 Roth C, Ferbert A. The posterior reversible encephalopathy syndrom: what's certain, what's new? Pract Neurol 2011; 11: 136-144

3 McKinney A, Short J, Truwit C et al. Posterior Reversible Encephalopathy Syndrome: Incidence of Atypical Regions of Involvement and Imaging Findings. AJR 2007; 189: 904-912

4 Bartynski W. Posterior Reversible Encephalopathy Syndrome, Part: Controversies Sourrounding pathophysiology of Vasogenic Edema. Am J Neuroradiol 2008; 29: 1043-1049

5 Golombeck S, Wessig C, Monoranu C-M et al. Fatal atypical reversible posterior leukencephalopathy syndrom: a case report. J Med Case Reports 2013; 7: 14

6 Lee V, Temes R, John S. Posterior reversible leukencephalopathy syndrome presenting with global cerebral Edema and Herniation. Neurocrit Care 2013; 18: 81-83

7 Facchini A, Magnomi S, Civelli V et al. Refractory intracranial Hypertension in posterior reversible encephalopathy Syndrome. Neurocrit Care 2013 [Epub ahead of print]

8 Roth C, Ferbert A. Posterior reversible encephalopathy syndrom: is there a difference between pregnant and non-pregnant patients? Eur Neurol 2009; 62: 142-148

9 Lee V, Eelco F, Manno E et al. Clinical Spectrum of reversible posterior Leukencephalopathy Syndrome“. Arch Neurol 2008; 65: 205-210

10 Korn A, Ernemann U, Nägele $T$ et al. „Posteriore Reversible Leukenzephalopathie“ Fortschr Röntgenstr 2010; 182: 377-380

11 Casey S, Sampaio R, Michel E et al. Posterior Reversible Encephalopathy Syndrome: Utility of Fluid-attenuated Inversion Recovery MR Imaging in the Detection of Cortical and Subcortical Lesions". Am J Neuroradiol 2000; 21: 1199-1206

12 Hefzy H, Bartynski W, Boardman J et al. Hemorrhage in Posterior Reversible Encephalopathy Syndrome: Imaging and Clinical Features. AM J Neuroradiol 2009; 30: 1371-1379

13 Kozak O, Wijdicks E, Manno E et al. Status epilepticus as initial manifestation of posterior reversible encephalopathy syndrome. Neurology 2007; 69: 894-897

14 Strandgaard S, Mackenzie E, Sengupta D et al. Upper Limit of the Autoregulation of Cerebral Blood Flow in the Baboon. Circ Res 1974; 34: $435-440$ 